\title{
Geoprocessamento Aplicado ao Estudo do ICH - Índice de Carência Habitacional para o Município de São José dos Campos a partir dos censos demográficos de 2000 e 2010
}

\author{
Thamy Barbara Gioia* \\ Mirian Vizintim Fernandes Barros**
}

\section{Resumo}

O ICH - Índice de Carência Habitacional, modelo criado pelo IPPUR - Instituto de Pesquisa e Planejamento Urbano e Regional da Universidade Federal do Rio de Janeiro, foi elaborado com o intuito de analisar a carência habitacional a partir de aspectos inerentes ao saneamento básico. Os resultados obtidos por meio deste modelo de análise permitem diagnosticar deficiências e auxiliar no planejamento para gestão adequada dos serviços de saneamento básico nos municípios. Verificando os índices calculados para as regiões metropolitanas de São Paulo, observouse dados extremamente positivos, diferente dos resultados apresentado pelos municípios das demais regiões do Brasil, fato que despertou nossa atenção quanto as possíveis variáveis motivadoras desta particularidade na região. Desta forma, o objetivo deste trabalho foi utilizar técnicas e ferramentas de geoprocessamento para calcular o ICH para o município de São Jose dos Campos-SP nos anos 2000 e 2010. Para isto os dados referentes ao censo IBGE foram espacializados e analisados a partir de ferramentas disponíveis no software ArcGIS 10. Os cenários resultantes do modelo permitiram classificar o município

Geógrafa e Especialista em Gestão Ambiental para Municípios (thamygioia@gmail.com).

** Doutora em Geografia Física (vizintim@uel.br).

Geosul, Florianópolis, v. 29, n. 57, p 131-156, jan./jun. 2014 
GIOIA, T.B. \& BARROS, M.V.F. Geoprocessamento aplicado ao estudo...

de São José dos Campos quanto a sua carência habitacional, além de proporcionar perspectivas para novas análises.

Palavras-chave: ICH; Planejamento urbano; Saneamento básico; Geoprocessamento.

Geoprocessing applied to the study of Index of Housing Deprivation for the city of São Jose dos Campos - SP from demographic census of 2000 e 2010

\begin{abstract}
The ICH - Índice de Carência Habitacional (Index of Housing Deprivation), model created by IPPUR - Institute for Research and Urban and Regional Planning, Federal University of Rio de Janeiro was elaborate with intention the analyze the shortage housing from the aspects inherent to basic sanitation. The results obtained by this model analysis allows diagnose deficiencies and auxiliary planning for proper management of basic sanitation services in the cities. Checking the indices calculated to the metropolitan region of São Paulo, observed extremely positive data, different from the results presented by cities in the other regions of Brazil, fact that aroused our attention as the possible variables motivating this particular view in the southeast region. So, the objective of this work was using techniques and geoprocessing tools to calculate the ICH to São Jose dos Campos - SP in years 2000 and 2010. For this the datas related for the census IBGE were spatialized and analyzed from tools available in the software ArcGis 10. The scenarios resulting the model allowed to classify the city of São Jose dos Campos as its shortage housing, beyond providing perspectives for new analysis.
\end{abstract}

Key words: ICH; Urban planning; Basic sanitation; Geoprocessing 
GIOIA, T.B. \& BARROS, M.V.F. Geoprocessamento aplicado ao estudo...

Introdução

O uso de técnicas e ferramentas de geoprocessamento aplicado ao planejamento e a gestão do território tem crescido nas últimas décadas. A possibilidade de armazenar dados e gerenciálos, como visualizá-los no espaço geográfico facilitam, e mesmo otimiza, o planejamento e gestão das atividades.

O ICH - Índice de Carência Habitacional, elaborado pelo Instituto de Pesquisa e Planejamento Urbano e Regional - IPPUR da Universidade Federal do Rio de Janeiro, indica o grau de carência habitacional de uma população calculado a partir da observação da qualidade dos serviços de saneamento básico disponíveis para os domicílios.

Os serviços de saneamento básico são divididos em quatro eixos: abastecimento de água, esgotamento sanitário, coleta e tratamento de resíduos sólidos e drenagem das águas pluviais. Os resultados obtidos através dos cálculos, em conjunto com sua respectiva espacialização, permitem tanto diagnosticar as deficiências em relação à disponibilização destes serviços à população, como direcionar o planejamento para sanar as deficiências levantadas (PLHIS DE IBIPORA-PR, 2011).

Observando o site oficial de difusão de dados referentes ao ICH das principais regiões metropolitanas do Brasil (OBSERVATÓRIO DA METROPOLES, 2013), verificou-se que os municípios pertencentes às regiões metropolitanas do Estado de São Paulo, apresentam valores extremamente positivos de $\mathrm{ICH}$, além de certa uniformidade nos resultados. Tal condição difere-se das demais regiões do Brasil, com exceção da região sul.

Desta forma, este trabalho teve por objetivo utilizar técnicas e ferramentas de geoprocessamento para o cálculo e análise do ICH do Município de São Jose dos Campos, SP nos anos 2000 e 2010 o qual poderá contribuir para os planos de ordenamento territorial.

Para atingir este objetivo, o trabalho foi divido em duas etapas: pesquisa de dados e produção de novas informações em ambiente SIG. A primeira refere-se ao levantamento dos dados dos censos IBGE- 
GIOIA, T.B. \& BARROS, M.V.F. Geoprocessamento aplicado ao estudo...

Instituto Brasileiro de Geografia e Estatística 2000 e 2010, e da malha digital para o município de São Jose dos Campos e a segunda, à elaboração de Banco de Dados a partir da união destas duas bases incluindo os resultados dos cálculos de ICH por Setor Censitário.

Espera-se que este trabalho possa contribuir para novas discussões a respeito da aplicação de ferramentas de geoprocessamento para produção e análise de dados espaciais, buscando contribuir para melhoria no planejamento e gestão do território no que diz respeito à carência habitacional.

\section{Aspectos conceituais}

\section{$\mathrm{O} \mathrm{ICH}$ - índice de carência habitacional}

O conceito de carência habitacional contempla dois segmentos distintos: o déficit e a inadequação habitacional. De acordo com o Ministério das Cidades (2007), estes se referem respectivamente ao estoque disponível de moradias e as moradias que não proporcionam a seus moradores condições desejáveis de habitabilidade, o que não implica, contudo, na necessidade de construção de novos domicílios.

Podem ser consideradas moradias em condições de inadequação habitacional os domicílios carentes de infraestrutura (ao menos deficientes em algum serviço básico de atendimento público: água, energia elétrica, esgotamento sanitário), com adensamento excessivo de moradores, com problemas de ordem fundiária, com alto grau de depreciação ou sem sanitário no interior da residência.

$\mathrm{O} \mathrm{ICH}$, desenvolvido pela equipe de pesquisa do observatório de Políticas Urbanas e Gestão Municipal do IPPUR foi criado no intuito de com os dados mais atuais disponíveis, fornecer um quadro sobre a carência habitacional dos municípios brasileiros, a partir de aspectos relacionados ao saneamento básico. Neste caso, o modelo utiliza de dados secundários obtidos pelos censos do IBGE.

Além disto, vale destacar que tal metodologia é empregada comumente na elaboração dos Planos de Habitação de Interesse Social 
GIOIA, T.B. \& BARROS, M.V.F. Geoprocessamento aplicado ao estudo...

(PLHIS) por recomendação do Ministério das Cidades. O PLHIS é um dos instrumentos de implantação do Sistema Nacional de Habitação de Interesse Social que tem por objetivo planejar as ações no setor habitacional de forma articulada (MINISTÉRIO DAS CIDADES, 2009). Este Plano consiste basicamente na elaboração do diagnostico do setor habitacional de um município apresentando um levantamento das necessidades habitacionais existentes e elaborando posteriormente a estratégia para minimização destas necessidades e deficiências.

Apenas para citar alguns dos trabalhos já desenvolvidos com a utilização do $\mathrm{ICH}$, destacam-se: a Política de Habitação de Interesse Social do município de Natal - RN, Porto Alegre - RS, Aparecida de Goiânia - GO, Paranavaí -PR e Ibiporã - PR.

Para o cálculo do $\mathrm{ICH}$, são observados primeiramente, os domicílios particulares permanentes enquadrados em categorias de inadequação quanto saneamento básico. $\mathrm{Na}$

Tabela 1 é apresentado as variáveis consideradas adequadas e inadequadas, de acordo com o censo IBGE.

Tabela 1: Matriz de definição de adequação e inadequação das variáveis do IBGE com base no censo 2000.

\begin{tabular}{|c|c|c|c|c|}
\hline \multicolumn{5}{|c|}{ Condição dos domicílios quanto ao tipo de situação da infraestrutura } \\
\hline $\begin{array}{c}\text { Rede geral com } \\
\text { canalização }\end{array}$ & $\begin{array}{c}\text { Rede geral com } \\
\text { canalização só } \\
\text { no terreno }\end{array}$ & $\begin{array}{c}\text { Poço com } \\
\text { canalização }\end{array}$ & $\begin{array}{c}\text { Poço com } \\
\text { canalização só no } \\
\text { terreno }\end{array}$ & $\begin{array}{c}\text { Poço sem } \\
\text { canalização } \\
\text { Outro }\end{array}$ \\
\hline ADEQUADO & ADEQUADO & ADEQUADO & INADEQUADO & INADEQUADO \\
\hline
\end{tabular}

\begin{tabular}{|c|c|c|c|c|}
\hline \multicolumn{5}{|c|}{ Esgotamento Sanitário } \\
\hline Rede Geral & Fossa Séptica & Fossa Rudimentar & $\begin{array}{c}\text { Vala Negra } \\
\text { Rio, lago ou mar }\end{array}$ & $\begin{array}{c}\text { Sem banheiro ou } \\
\text { sanitário } \\
\text { Outros }\end{array}$ \\
\hline ADEQUADO & ADEQUADO & INADEQUADO & INADEQUADO & INADEQUADO \\
\hline \multicolumn{5}{|c|}{ Disposição de Resíduos Sólidos } \\
\hline Coletado direto & $\begin{array}{c}\text { Coletado } \\
\text { indireto }\end{array}$ & Queimado & Enterrado & $\begin{array}{c}\text { Jogado no terreno } \\
\text { Joga em rio, lago } \\
\text { ou mar }\end{array}$ \\
\hline ADEQUADO & ADEQUADO & INADEQUADO & INADEQUADO & INADEQUADO \\
\hline
\end{tabular}

Fonte: IPPUR, 2003.

Adaptado por: Thamy Barbara Gioia 
GIOIA, T.B. \& BARROS, M.V.F. Geoprocessamento aplicado ao estudo...

$\mathrm{Na}$ categoria adequada estão os domicílios com abastecimento de água por rede geral de abastecimento e ou canalizadas, com esgotamento sanitário em rede geral ou em fossas sépticas e coleta de resíduos sólidos por serviço público de coleta. Sendo consideradas inadequadas todas as demais categorias disponíveis não enquadradas nas categorias acima descritas.

Após obtenção destes valores, as variáveis consideradas inadequadas são somadas e posteriormente transformadas em porcentagem, conforme exemplificado na Equação 1, onde Di representa o total de domicílios e i corresponde a cada variação do indicador.

$$
\mathrm{Di}=\left[\left(\frac{1}{Y}\right) \sum v i\right] \quad \text { Equação } 1
$$

A partir do cálculo das porcentagens procede-se com o cálculo do $\mathrm{ICH}$. O modelo matemático do $\mathrm{ICH}$ é apresentado na Equação 2, onde a variável vo (valor observado) representa o percentual de domicílios com pior índice de atendimento nos seguintes eixos do saneamento básico, ou setor de serviço (ss): abastecimento de água, esgotamento sanitário e disposição dos resíduos sólidos (POLIDORO et al, 2009).

$$
I C H_{s s}=v o-\left(\frac{100}{0-100}\right) \quad \text { Equação 2 }
$$

O resultado deste cálculo indicará a percentagem de domicílios em situação inadequada em cada eixo. Desta, forma quanto maior a porcentagem de domicílios enquadrados em condições inadequadas piores serão os índices de carência habitacional.

A partir dos resultados obtidos do $\mathrm{ICH}$ por eixo de saneamento é possível calcular a média ponderada conforme a Equação 3 e a 
GIOIA, T.B. \& BARROS, M.V.F. Geoprocessamento aplicado ao estudo...

Tabela 2, no qual o ICH é a media ponderada do índice, $\mathrm{ICH}_{\mathrm{ss}}$ é o índice de carência para cada setor de serviço $i$ e p é o peso de cada setor.

$$
I C H=\sum_{I}^{i} I C H_{S S} i(p)
$$

Equação 3

Tabela 2: Relação de pesos por setor de serviço para obtenção da média ponderada.

\begin{tabular}{lc}
\multicolumn{1}{c}{ ICH } & PESO \\
\hline ÁGUA & 3 \\
\hline ESGOTAMENTO SANITARIO & 2 \\
\hline RESIDUOS URBANOS & 1 \\
\hline
\end{tabular}

Fonte: OBSERVATORIO DAS METROPOLES, 2012.

Os resultados da média ponderada são classificados segundo as faixas apresentadas na Tabela 3 , a seguir.

Tabela 3: Categorias de carência Habitacional a partir de faixas de classificação.

\begin{tabular}{lc}
\multicolumn{1}{c}{ CATEGORIA } & $\begin{array}{c}\text { FAIXA DE } \\
\text { CLASSIFICAÇÃO }\end{array}$ \\
\hline EXTREMO ÍNDICE DE CARÊNCIA HABITACIONAL & 0 a 0,5 \\
\hline ALTO ÍNDICE DE CARÊNCIA HABITACIONAL & 0,5 a 0,8 \\
\hline BAIXO ÍNDICE DE CARÊNCIA HABITACIONAL & 0,8 a 1 \\
\hline
\end{tabular}

Fonte: OBSERVATORIO DAS METROPOLES, 2012.

Atualmente o Observatório das Metrópoles em conjunto com o IPPUR e o METRODATA, disponibilizam os resultados de ICH calculados para as principais regiões metropolitanas do Brasil, capitais e municípios com mais de 500 mil habitantes. $\mathrm{O}$ banco de 
GIOIA, T.B. \& BARROS, M.V.F. Geoprocessamento aplicado ao estudo...

dados pode ser consultado no site oficial do grupo de estudos: http://www.observatoriodasmetropoles.ufrj.br/metrodata/ich/.

\section{Materiais e método}

\section{Área de estudo}

O município de São José dos Campos está localizado no interior do Estado de São Paulo, mesorregião do Vale do Paraíba Paulista (Figura 1), coordenadas de latitude $23^{\circ} 10^{\prime} 47^{\prime \prime} \mathrm{S}$ e longitude 4553'14" O. Atualmente possui uma população de 629.921 habitantes (IBGE, 2010), considerado em 2010 o sétimo município mais populoso no estado de São Paulo.

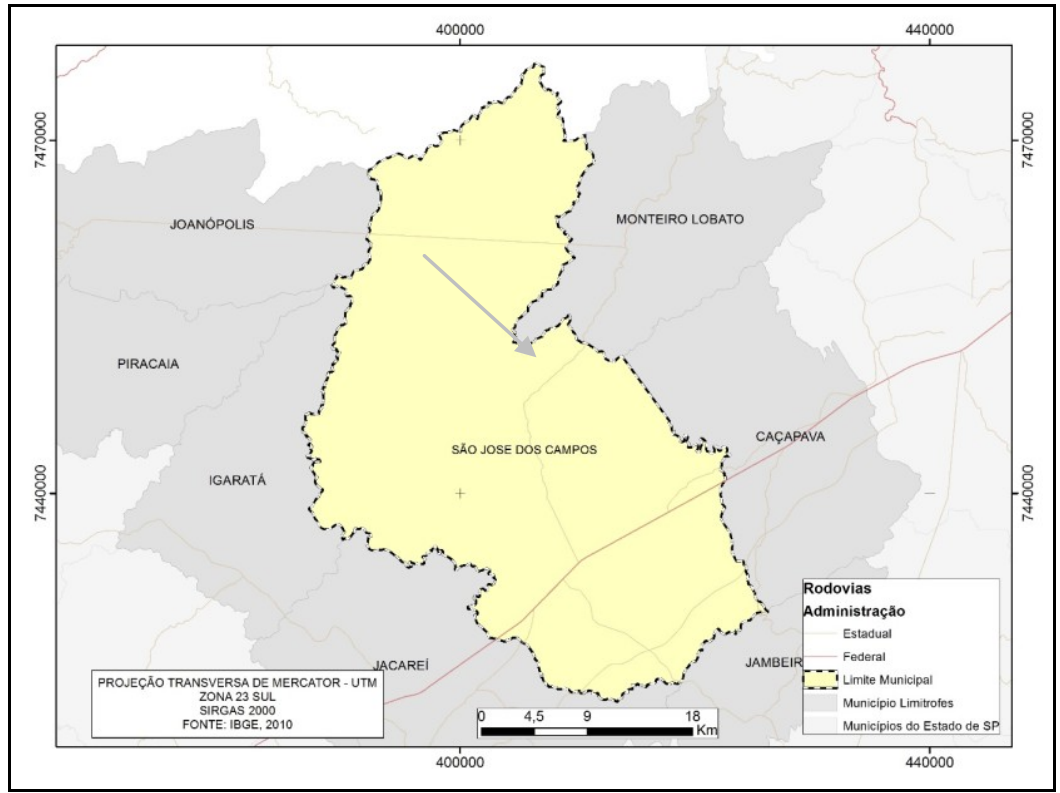

Figura 1: Localização do municipio de São José dos Campos.

Projeção: Transversa de Mercator - UTM, Zona 23 Sul, SIRGAS 2000.

Fonte: IBGE, 2010. 
GIOIA, T.B. \& BARROS, M.V.F. Geoprocessamento aplicado ao estudo...

\section{Dados e ferramentas}

O ICH para o Município de São Jose dos Campos-SP foi calculado separadamente por setor censitário, que corresponde a menor unidade territorial formada por área continua integralmente contida em área urbana ou rural (IBGE, 2010).

Para realização deste estudo foram utilizados dados referentes aos censos dos anos de 2000 e 2010 disponíveis em formato xls e malhas digitais em formato shapefile (*.shp), disponíveis no site oficial do IBGE. Estes dados foram trabalhados em ambiente SIG por meio de ferramentas disponíveis no software ArcGIS 10.0.

\section{Método}

A metodologia empregada para realização do trabalho pode ser observada no Modelo OMT-G - Object Modeling Technique for Geographic Applications, da Figura 2. Os processamentos serão explicados com detalhe a seguir.

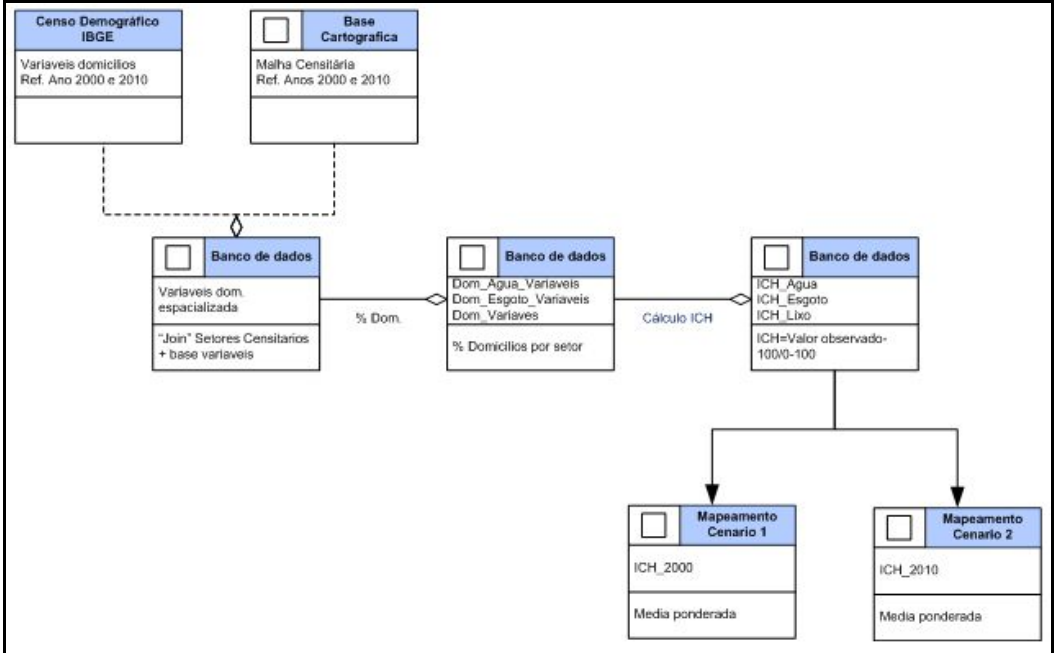

Figura 2: - Modelo OMT-G do Projeto referente a analise de ICH para o município de São Jose dos Campos - SP. 
GIOIA, T.B. \& BARROS, M.V.F. Geoprocessamento aplicado ao estudo...

Para espacialização das variáveis referentes aos domicílios procedeu-se com a concatenação da base de dados em formato .xls e da malha censitária em formato shapefile através da ferramenta joining.

Para efetivação da concatenação é necessário que os arquivos possuam uma "chave" comum, neste caso, foram utilizados os Códigos Alfanuméricos dos setores censitários para o município de São Jose dos Campos. Este procedimento foi realizado para as duas bases censitárias, 2000 e 2010.

No caso da malha censitária de 2000, foram necessárias algumas adequações tendo em vista que a mesma encontrava-se dividida em dois arquivos shapefile, um referente à área urbana e outra para área rural. Desta forma as malhas foram unidas em um único shape. Os setores sobrepostos, no caso dois, que representavam a área urbana na malha censitária rural, foram retirados da tabela de atributos para que não ocorresse a sobreposição de valores.

Após a concatenação das bases (malha digital e informações do censo) procedeu-se à elaboração dos dados referentes ao $\mathrm{ICH}$ para os dois períodos. Para a construção destes dados utilizou-se a porcentagem de domicílios enquadrados na categoria inadequados (

Tabela 1) quanto aos eixos de saneamento - abastecimento de água, esgotamento sanitário e disposição de resíduos sólidos.

As variáveis utilizadas neste procedimento são diferentes para o ano 2000 e 2010, tendo sido necessário a elaboração de dois modelos matemáticos distintos paras as análises. As adaptações referem-se às variáveis utilizadas para os cálculos de porcentagem e podem ser visualizadas na 
GIOIA, T.B. \& BARROS, M.V.F. Geoprocessamento aplicado ao estudo...

Tabela 4. 
GIOIA, T.B. \& BARROS, M.V.F. Geoprocessamento aplicado ao estudo...

Tabela 4: Variáveis consideradas inadequadas quanto ao atendimento pelos serviços, ano 2000 e 2010.

\begin{tabular}{|c|c|}
\hline CENSO 2000 & CENSO 2010 \\
\hline \multicolumn{2}{|c|}{ VARIÁVEIS INADEQUADAS } \\
\hline \multicolumn{2}{|c|}{ Categoria - Água } \\
\hline V023, V024, V025 & V013, V014 e V015 \\
\hline \multicolumn{2}{|c|}{ Categoria - Esgotamento Sanitário } \\
\hline V032, V033, V034, V035, V036 & V019, V020, V021, V022, V023 \\
\hline \multicolumn{2}{|c|}{ Categoria - Resíduos Sólidos } \\
\hline
\end{tabular}

Fonte: IBGE, 2010.

Adaptado por: Thamy Barbara Gioia

Dando sequência à produção dos dados, foram inseridas três novas colunas na tabela de atributos do shape referente ao censo 2000 e 2010 denominadas, porce_agua, porce_esgo e porce_lixo. A Figura 3 representa o processo de implantação da equação na tabela de atributos e os respectivos resultados.

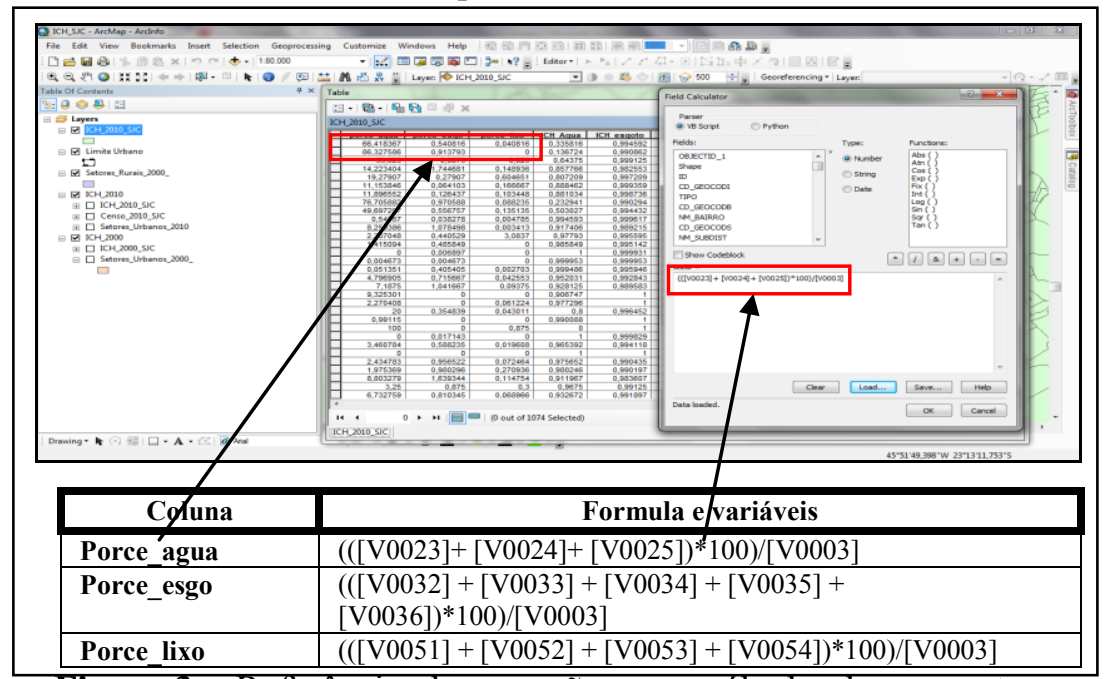

Figura 3: Referência de equação para cálculo da porcentagem dos domicílios classificados em categoria inadequada quanto aos serviços de saneamento básico. 
GIOIA, T.B. \& BARROS, M.V.F. Geoprocessamento aplicado ao estudo...

Com a porcentagem calculada e inserida na tabela de atributos procedeu-se com o cálculo do $\mathrm{ICH}$ por setores, como pode ser observado na Figura 4.

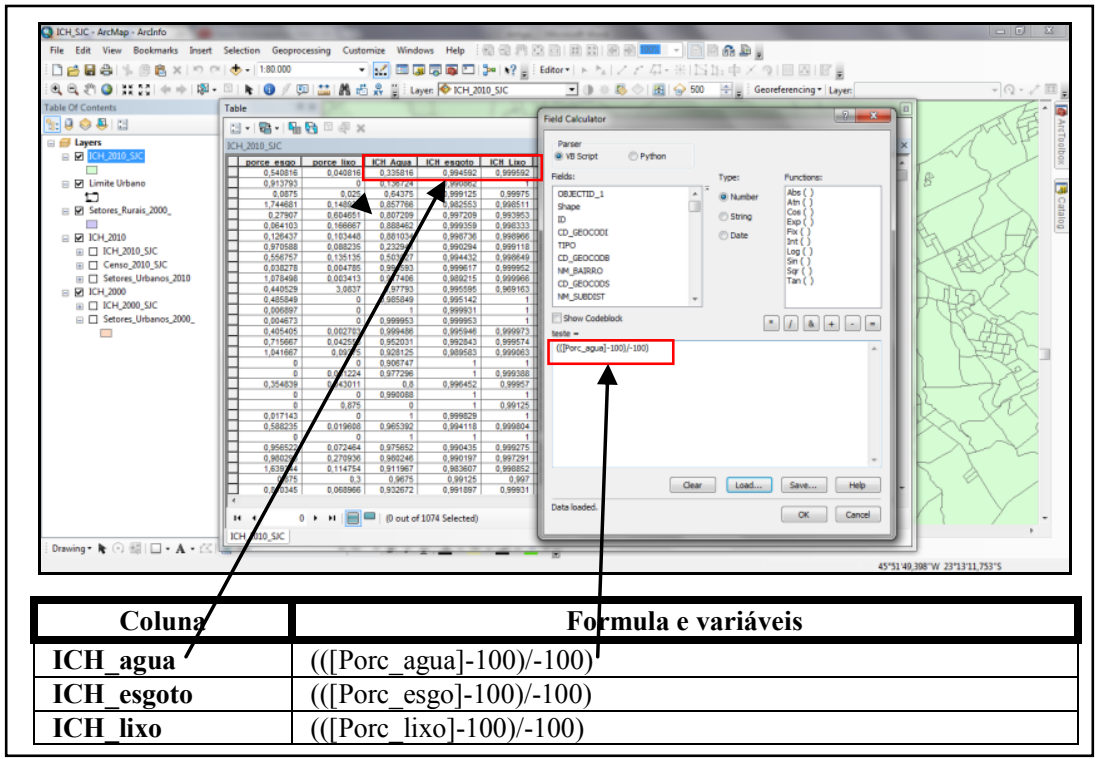

Figura 4: Referência de equação para cálculo do ICH por setor de serviços de saneamento básico.

Para finalizar o procedimento, calculou-se o ICH geral por setor censitário através da média ponderada (Figura 4). 
GIOIA, T.B. \& BARROS, M.V.F. Geoprocessamento aplicado ao estudo...

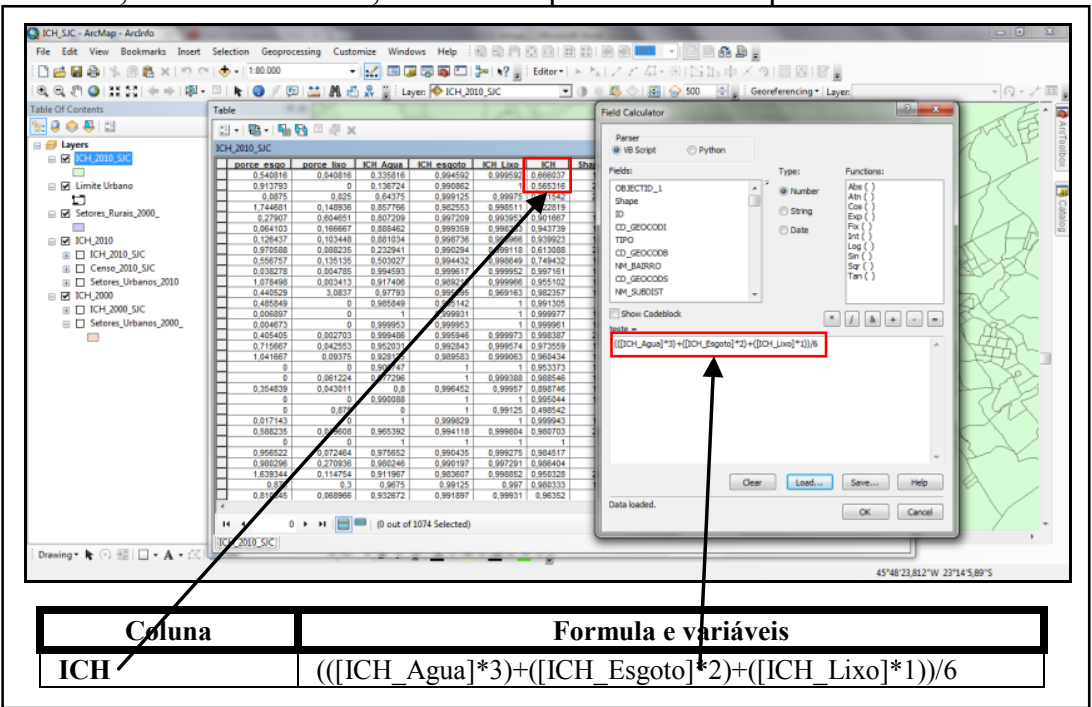

Figura 5: Referência de equação para cálculo do ICH médio por setor de serviços de saneamento básico.

Os resultados do ICH foram classificados conforme a Tabela 3 para cada ano, setor e média total para cada ano.

\section{Resultados}

Os dados do censo IBGE 2010 revelam que no ano de 2000 o município de São José dos Campos apresentava um total de 758 setores de recenseamento, dos quais 20 estavam localizados na área rural. Em 2010 esse valor aumenta para 1054 setores, dos quais 33 estavam em área rural. Quanto ao total de domicílios, o censo apresenta um total de 144.298 em 2000 e de 206.434 em 2010, o que representa um aumento de aproximadamente $70 \%$.

$\mathrm{O} \mathrm{ICH}$ do município revelou valores praticamente iguais para os dois anos: de 0.96 em 2000 e de 0.97 em 2010, valores que 
GIOIA, T.B. \& BARROS, M.V.F. Geoprocessamento aplicado ao estudo...

classificam o município dentro da faixa "Baixo índice de Carência Habitacional" (Figura 6).

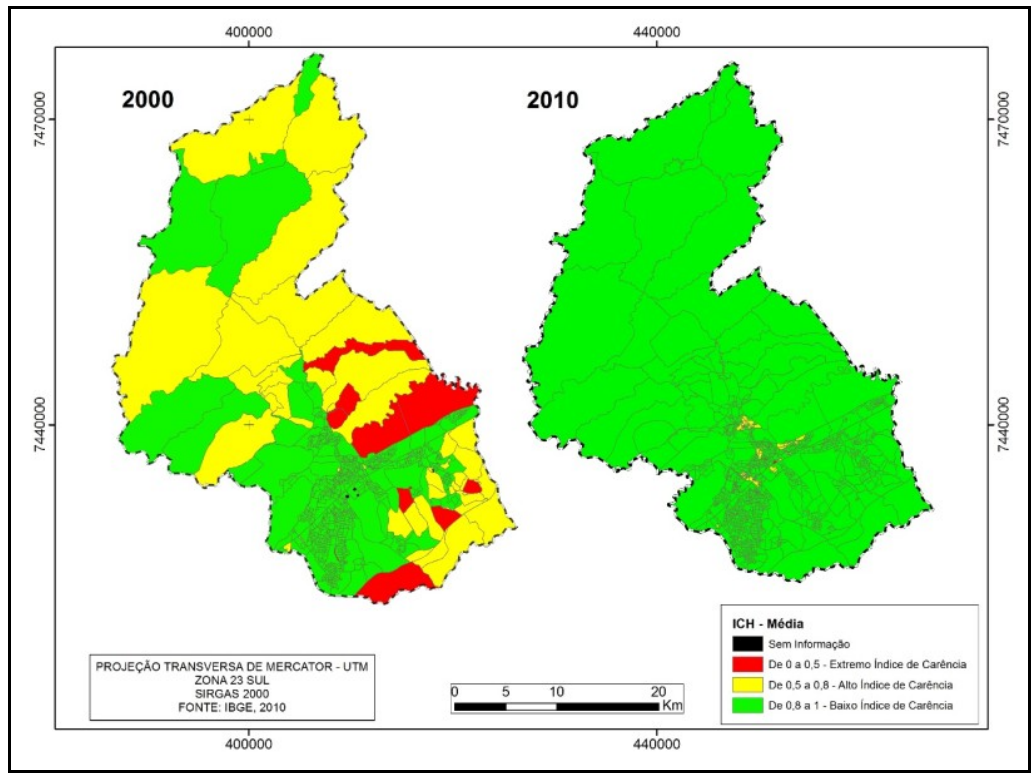

Figura 6: ICH Médio para o município de São Jose dos Campos para o ano 2000 e 2010.

Fonte: Base cartográfica IBGE, 2010.

Este resultado contrasta com o que se esperaria para o percentual de crescimento populacional do município no período, e indica que houve investimento no saneamento básico.

No entanto, se considerados apenas os dados dos domicílios da área rural em 2000, o município seria classificado em "Alto índice de Carência" habitacional, com destaque para o eixo de esgotamento sanitário para o qual se obteve um ICH de 0,44 . Já em 2010 este valor muda significativamente, ao atingir o valor de um (1) (Tabela 5). 
GIOIA, T.B. \& BARROS, M.V.F. Geoprocessamento aplicado ao estudo...

Tabela 5: ICH para o município de São Jose dos Campos. Anos 2000 e 2010

\begin{tabular}{lccccc}
\hline \multicolumn{1}{c}{ Ano } & $\begin{array}{c}\text { ICH } \\
\text { Água }\end{array}$ & $\begin{array}{c}\text { ICH } \\
\text { Esgoto }\end{array}$ & $\begin{array}{c}\text { ICH } \\
\text { Lixo }\end{array}$ & $\begin{array}{c}\text { ICH } \\
\text { Total }\end{array}$ & Situação \\
\hline 2000 - urbano & 098 & 0,94 & 0,99 & 0,97 & Baixo Índice de Carência \\
\hline 2000 - rural & 0,87 & 0,44 & 0,72 & 0,69 & Alto índice de Carência \\
\hline 2010 - urbano & 0,95 & 0,99 & 0,99 & 0,97 & Baixo índice de Carência \\
\hline 2010 - rural & 0,99 & 1 & 0,99 & 0,99 & Baixo índice de Carência \\
\hline
\end{tabular}

Fonte: IBGE (2000 e 2010).

Em 2000, os melhores valores do ICH se concentraram principalmente na área urbana, região central do município, enquanto os piores na região leste do município, nas proximidades dos bairros Jardim Helena, Bom Retiro, Campos de São José e Residencial São Francisco devido principalmente as condições de esgotamento sanitário e abastecimento de agua categorizadas pelo censo (Figura 7).

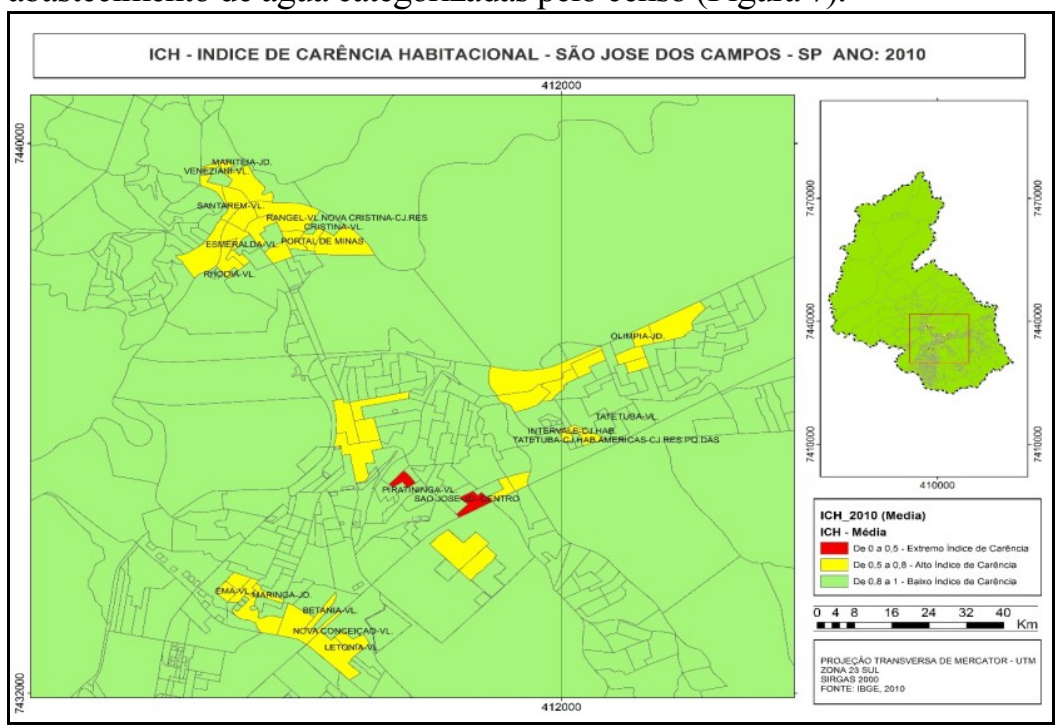

Figura 7: ICH Médio ano de 2010. Destaque para as áreas correspondentes aos piores índices identificados.

Fonte: Base cartográfica IBGE, 2010. 
GIOIA, T.B. \& BARROS, M.V.F. Geoprocessamento aplicado ao estudo...

No eixo abastecimento de água, os piores valores se concentram nas mesmas regiões identificadas para o ICH médio de 2000, entretanto, no geral os valores são bem positivos, principalmente pelos resultados gerados para o ano de 2010, classificando o município em "baixo índice de carência” (Figura 8).

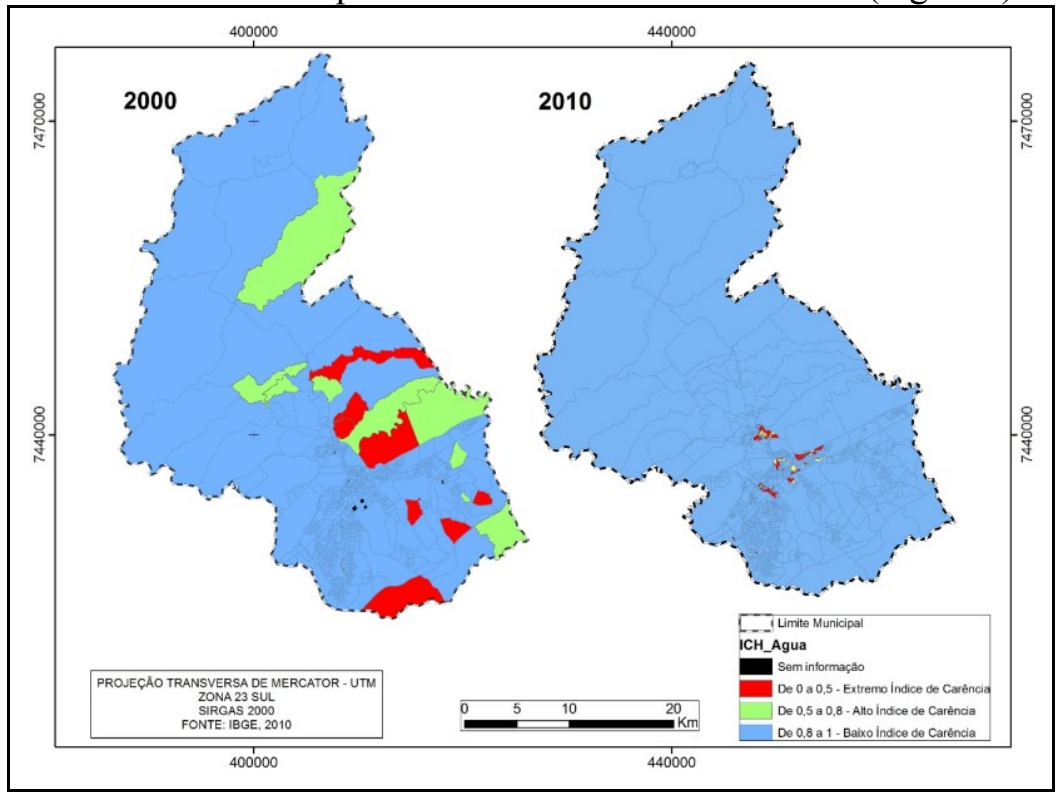

Figura 8: ICH Água para o município de São Jose dos Campos para o ano 2000 e 2010.

Fonte: Base cartográfica IBGE, 2010.

No ano 2000 também foram observados altos índices de carência no setor de esgotamento sanitário e em proporção significativa: 49 setores censitários apresentaram valores abaixo de 0,5, a maior parte concentrada na área rural e áreas adjacentes ao centro urbano (Figura 9). Isto indica a precariedade no atendimento por este serviço, situação comum em áreas rurais, periféricas e de expansão urbana de muitos municípios brasileiros. 
GIOIA, T.B. \& BARROS, M.V.F. Geoprocessamento aplicado ao estudo...

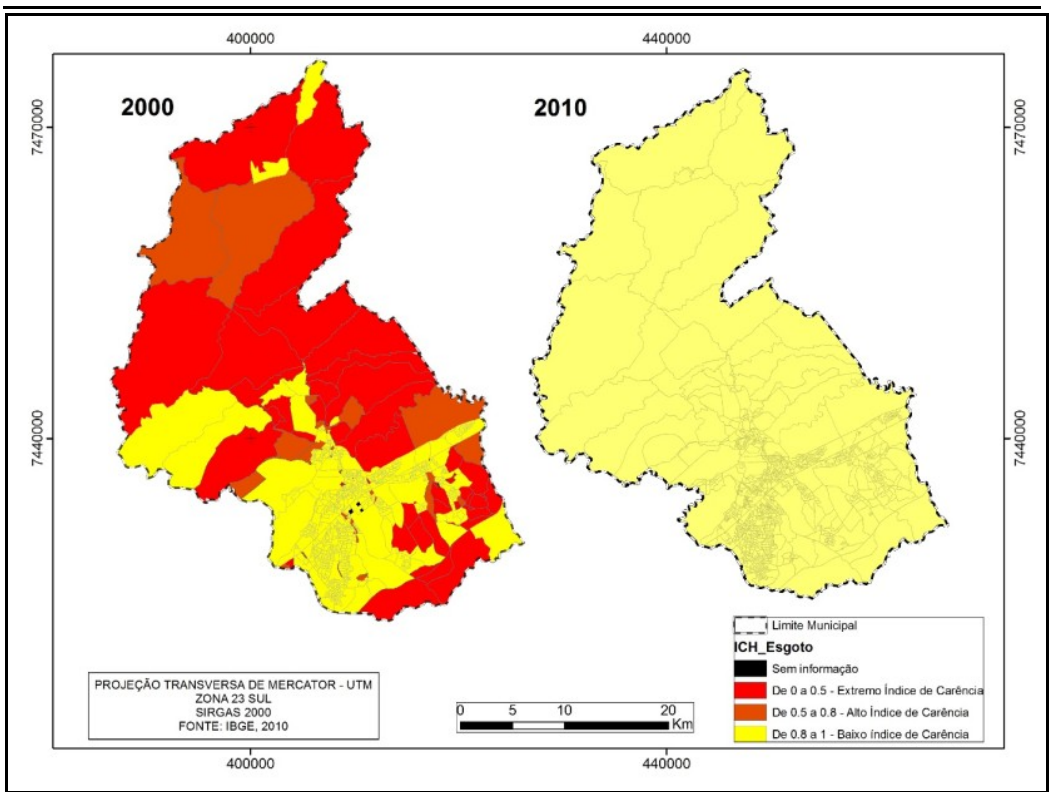

Figura 9: ICH Esgotamento Sanitário para o município de São Jose dos Campos para o ano 2000 e 2010

Fonte: Base cartográfica IBGE, 2010.

Em 2010, verifica-se uma melhora significativa nos valores quanto ao esgotamento sanitário (Figura 9). Boa parte deste aumento esta relacionado ao grande número de domicílios dispostos na categoria de esgotamento sanitário "fossa séptica", o que para fins de cálculo, é considerado categoria adequada de esgotamento sanitário. Conforme pode ser observado na 
GIOIA, T.B. \& BARROS, M.V.F. Geoprocessamento aplicado ao estudo...

Tabela 6, em 2010 aproximadamente $80 \%$ dos domicílios estavam ligados à rede pública de esgotamento sanitário, junto a $10 \%$ de domicílios registrados com fossas sépticas. 
GIOIA, T.B. \& BARROS, M.V.F. Geoprocessamento aplicado ao estudo...

Tabela 6: Variáveis de esgotamento sanitário pelos censos de 2000 e 2010

\begin{tabular}{ccccc}
\hline Ano & $\begin{array}{c}\text { Domicilios } \\
\text { Particulares } \\
\text { Permanentes }\end{array}$ & $\begin{array}{c}\text { Esgotamento } \\
\text { por rede de } \\
\text { coleta }\end{array}$ & Fossa Séptica & $\begin{array}{c}\text { Fossa } \\
\text { Rudimentar }\end{array}$ \\
\hline \multirow{2}{*}{2000} & 144.298 & 128.600 & 7.369 & 5.049 \\
\cline { 2 - 5 } & $100,00 \%$ & $89,12 \%$ & $5,11 \%$ & $3,50 \%$ \\
\hline \multirow{2}{*}{2010} & 206.434 & 163.126 & 20.957 & 12.647 \\
\cline { 2 - 5 } & $100,00 \%$ & $79,02 \%$ & $10,15 \%$ & $6,13 \%$ \\
\hline
\end{tabular}

Fonte: IBGE, 2010.

Já no eixo resíduos sólidos, observa-se que para o ano de 2000, os melhores índices concentram na área urbana do município, enquanto que os piores na área rural. Em 2010, apenas 6 setores censitários apresentaram valores abaixo de 0,5 , enquanto em 2000 o registro é de 13 setores, o que proporcionalmente representa uma melhoria neste eixo.

Conforme dados referentes aos dois censos (2000 e 2010) deve-se essa melhora a redução no número de domicílios enquadrados em categorias inadequadas de disposição de resíduos sólidos, como: queima na propriedade, enterrado na própria propriedade ou outra forma de disposição, em alguns dos setores censitários. 
GIOIA, T.B. \& BARROS, M.V.F. Geoprocessamento aplicado ao estudo...

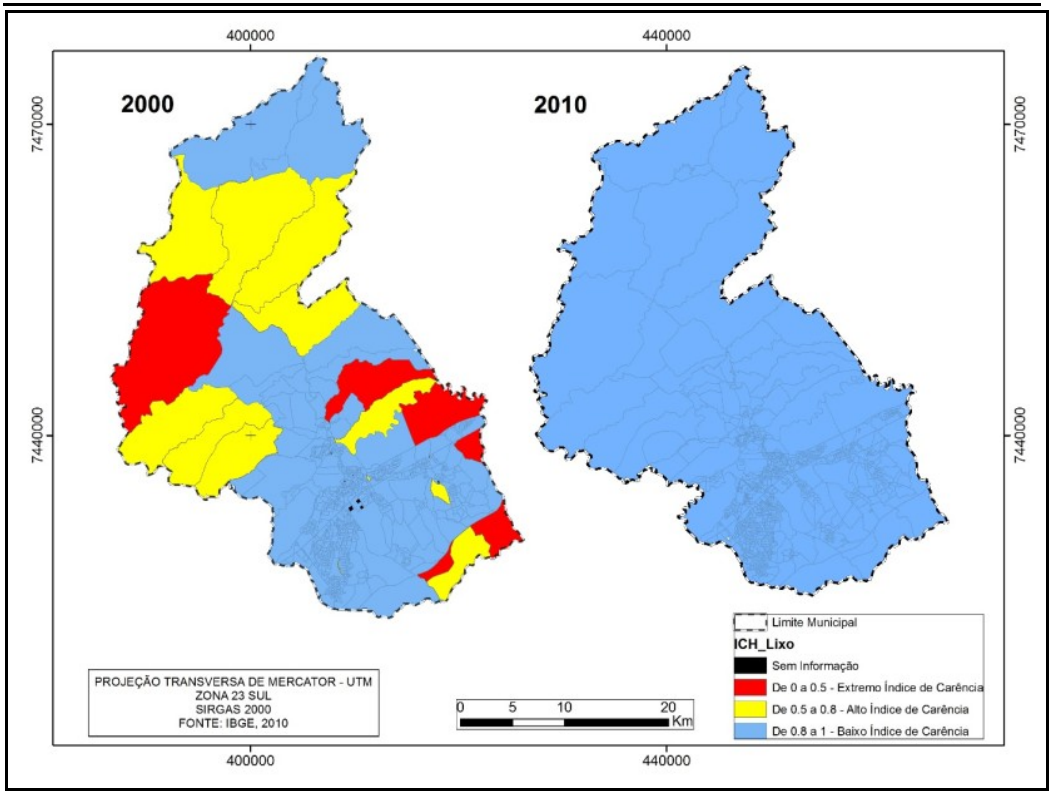

Figura 10: ICH Disposição de Resíduos Sólidos para o município de São Jose dos Campos para o ano 2000 e 2010.

Fonte: Base cartográfica IBGE, 2010.

Embora os resultados para o ano de 2010 sejam positivos, é necessário destacar que 45 setores censitários foram enquadrados na categoria "Alto Índice de Carência", todos localizados na área urbana do município, nas regiões da Vila Ema, Guaianazes, Maringá, Zelfa, Betânia, Nova Conceição, Letônia, Jardim Matarazzo, Vila Pedro, Conjunto Residencial Parque das Américas, Ismênia, Maracanã, Jardim Brasília, Jardim Olímpia, Veneziana, Jardim Maritéia, Vila Nossa Senhora das Graças, Alto da Ponte, Vila Santarém, Vila Machado, Jardim Anchieta, Jardim Ouro Preto, Vila Rangel, Jardim Nova Paulicéia, Vila Esmeralda, Santana, Nova Cristina, Vila Chiquinha, Portal de Minas, Jaci, Pasto Alto e Vila Carmo. 
GIOIA, T.B. \& BARROS, M.V.F. Geoprocessamento aplicado ao estudo...

Boa parte dos bairros citados como a Vila Ema, por exemplo, estão localizados em região nobre de São José dos Campos. Em parte deve-se os altos valores ao alto índice de domicílios enquadrados em categorias inadequadas de abastecimento de água.

Observando os dados em detalhe, verificou-se que este baixo índice de abastecimento de água por rede pública em alguns setores censitários localizados na região central do municipio contrasta com um alto índice de atendimento por rede pública de esgotamento sanitário, o que poderia induzir a um erro nos resultados e consequentemente nas análises, sabendo-se que no geral, o comum em áreas ocupadas e já consolidadas é a disponibilização de agua tratada por rede pública e posteriormente a coleta do esgotamento sanitário.

Após uma verificação cuidadosa do banco de dados identificou-se a seguinte situação: nesses setores censitários existe um número não desprezível de domicílios registrados na variável V015 - "Domicílios particulares permanentes com outra forma de abastecimento de água”.

Esta variável é utilizada para descrever a desconformidade no atendimento; um alto índice de domicílios enquadrados nesta categoria altera substancialmente $\mathrm{o}$ resultado final $\mathrm{e}$ consequentemente as análises a partir destas informações.

Estima-se cerca de 60 setores com alto índice de domicílios classificados em "outra forma de abastecimento de água" que não a enquadra em nenhuma das variáveis anteriores. De acordo com o IBGE (2010) entende-se por outra forma de abastecimento aquela que adquire água de fontes como poço ou nascente fora da propriedade, carro-pipa, água da chuva armazenada, rio, açude, lago ou igarapé ou outra forma diferente das descritas anteriormente.

Esta situação foi discutida com outros técnicos e pesquisadores, inclusive do IBGE, a conclusão em geral indica erro quanto ao levantamento de dados em campo, ou seja, na coleta dos dados realizada pelos recenseadores. 
GIOIA, T.B. \& BARROS, M.V.F. Geoprocessamento aplicado ao estudo...

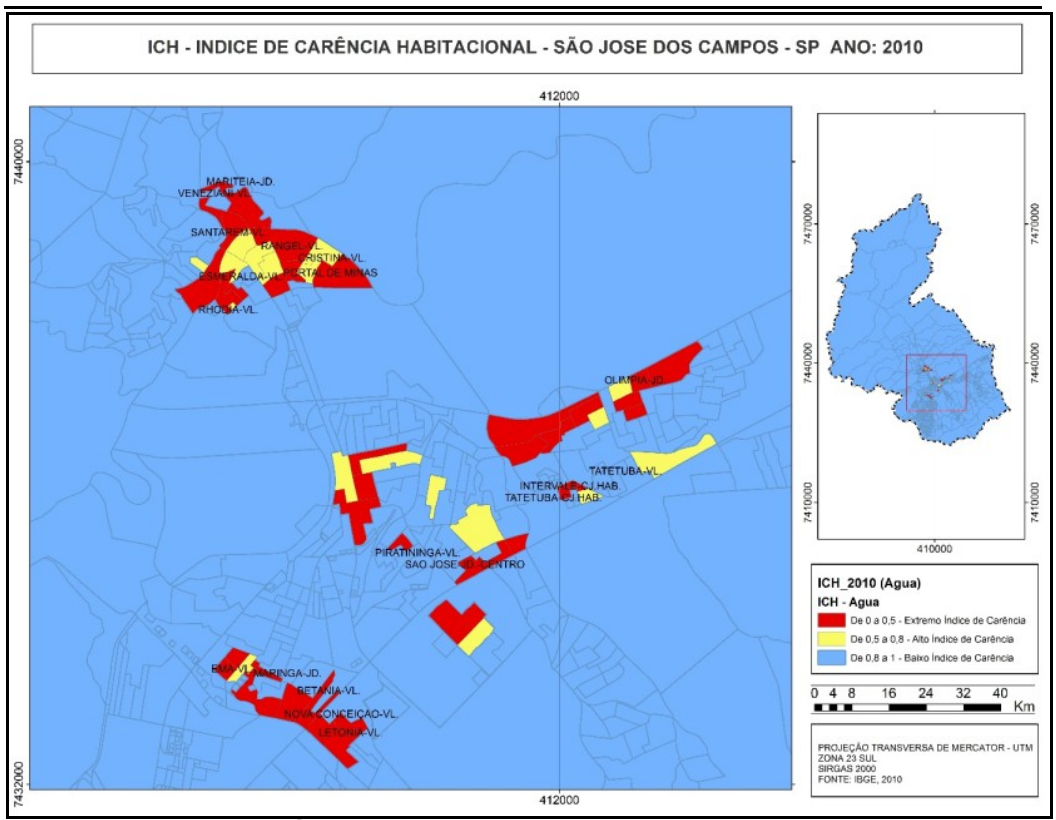

Figura 11: ICH Água no ano de 2010. Destaque para as áreas correspondentes aos piores índices identificados.

Fonte: Base cartográfica IBGE, 2010.

Na Figura 12 esta representado alguns dos setores censitários classificados nesta categoria, como alguns localizados no bairro Vila Ema com cerca de 300 os domicílios nestas condições. 
GIOIA, T.B. \& BARROS, M.V.F. Geoprocessamento aplicado ao estudo...

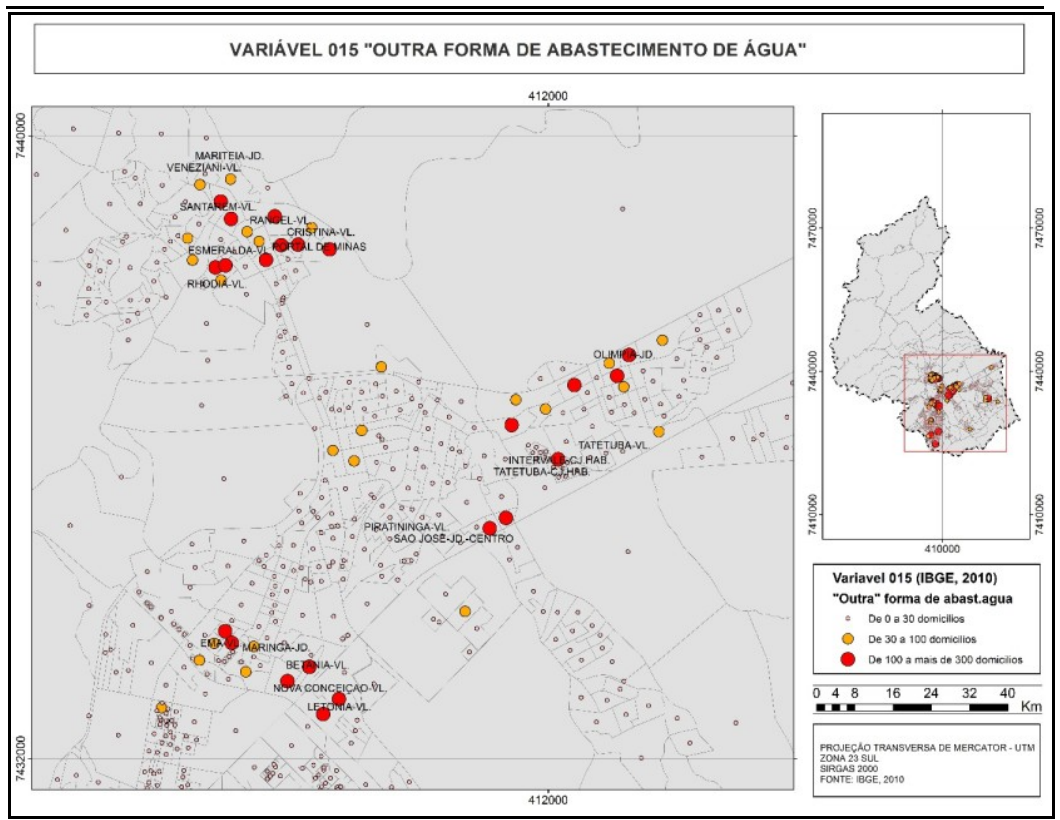

Figura 12: Espacialização da variável 015, censo de 2010 "Outra forma de abastecimento de água".

Fonte: Base cartográfica IBGE, 2010.

De acordo com dados do Plano Municipal de Saneamento Básico de São José dos Campos, o Município tem cobertura por redes de distribuição de água em 100\% de sua área regular urbanizada, excetuando-se apenas os loteamentos irregulares, Portanto se estima um índice de atendimento populacional próximo de $94 \%$, o que não condiz com os resultados obtidos através do modelo $\mathrm{ICH}$.

\section{Considerações finais}

Sabe-se que o censo demográfico é a mais complexa operação estatística realizada por um país, onde são investigadas diversas características dos domicílios e da população residente. Além disto, 
GIOIA, T.B. \& BARROS, M.V.F. Geoprocessamento aplicado ao estudo...

constituem fonte de referência para conhecimento das condições de vida da população e seus recortes territoriais (IBGE, 2010).

A metodologia utilizada para levantamento destes dados, hoje mais automatizada, consiste basicamente na aplicação de um questionário pré-definido, por recenseador treinado para este fim. Entretanto, as respostas obtidas junto ao morador, são dadas como corretas, não sendo obrigação do recenseador verificar se as condições descritas pelo morador representam realmente a realidade.

Um caso que gera grandes dúvidas nas respostas obtidas pelo censo diz respeito às fossas sépticas e rudimentares, nem todos detém o conhecimento quanto às diferenças inerentes a construção das mesmas.

Inconsistências como as levantadas no quesito abastecimento de água e esgotamento sanitário podem comprometer significativamente os resultados, tendo em vista que o ICH dependente diretamente das respostas obtidas pelo censo.

Além disto, vale ressaltar que o saneamento básico contempla quatro eixos de serviços - Abastecimento de água, esgotamento sanitário, coleta, transporte $r$ disposição de resíduos e drenagem das águas pluviais. $\mathrm{O}$ modelo proposto para o calculo de $\mathrm{ICH}$ desconsidera o setor de drenagem urbana. Desta forma, outras análises e pesquisas na perspectiva de inserir tal informação, poderia complementar e colaborar para resultados mais consistentes.

Desta forma, conclui-se que os resultados obtidos a partir do calculo de ICH para o município de São José dos Campos atende ao objetivo proposto, entretanto, ressalta-se a importância de ampliar a análise por intermédio de outras fontes de dados como: levantamentos em campo, entrevistas junto a técnicos da Prefeitura responsáveis pela gestão dos serviços além de entrevista junto à empresa responsável pela execução dos serviços de saneamento básico.

\section{Referencias bibliográficas}

BRASIL. Ministério das Cidades (2005) Lei 11.124, de 16 de junho de 2005: Dispõe sobre o Sistema Nacional de Habitação de 
GIOIA, T.B. \& BARROS, M.V.F. Geoprocessamento aplicado ao estudo...

Interesse Social - SNHIS, cria o Fundo Nacional de Habitação de Interesse Social - FNHIS e institui o Conselho Gestor do FNHIS.

CAMARA, Gilberto et al. Anatomia de Sistemas de Informação Geográfica. Disponível em: http://mtcm12.sid.inpe.br/col/sid.inpe.br/sergio/2004/10.07.13.36/doc/anato mia.pdf.Acesso em 21.Mar.2013.

FUNDAÇÃO JOÃO PINHEIRO. Déficit Habitacional no Brasil. Disponível em: http://www.fjp.mg.gov.br/ Acesso em 20.abr.2013.

GENOVEZ, Patricia C. et al. Medidas Territoriais de Desigualdade Social: Análise Espacial da Dinâmica de Exclusão/Inclusão Social em São Jose dos Campos-SP (1991-2000). Disponível em: http://biblioteca.cptec.inpe.br/. Acesso em 30.abr.2013.

IBGE - Instituto Brasileiro de Geografia e Estatística. Censos Demográficos 2000 e 2010. Disponível em: http://downloads.ibge. gov.br/downloads_estatisticas.htm. Acesso em 25.mar.2013.

IPPUR - Instituto de Pesquisa e Planejamento Urbano e Regional. ICH - Índice de Carência Habitacional. Disponível em < http://www.observatoriodasmetropoles.ufrj.br/metrodata/ich/index. html>. Acesso em 17. Mar.2013.

LEAO, Simone Zarpelon e TURKIENICZ, Benamy. Análise da Acessibilidade urbana para o planejamento da urbanização de interesse social. Disponível em: < http://biblioteca.cptec.inpe.br/> Acesso em 30.abr.2013.

MINISTÉRIO DAS CIDADES. Planos Locais de Habitação de Interesse Social. Brasília: 2009.

. Déficit Habitacional no Brasil. Brasília: 2007. 
GIOIA, T.B. \& BARROS, M.V.F. Geoprocessamento aplicado ao estudo...

OBSERVATÓRIO DAS METRÓPOLES. ICH - Índice de Carência Habitacional. Disponível em: < http://www.observatoriodasmetropoles.ufrj.br/metrodata/ich/>. Acesso 01. Abr.2013

POLIDORO, Mauricio; TAKEDA, Mariane Mayumi Garcia e BARROS, Omar Neto Fernandes. Mapeamento do Índice de Carência Habitacional na Região Metropolitana de Londrina PR. Disponível em: < http://www.geo.uel.br/didatico/omar/ modulo_b/a7.pdf $>$. Acesso em 19. Mar.2013.

PREFEITURA MUNICIPAL DE IBIPORA - PR. PLHIS - Plano Local de Habitação de Interesse Social, 2013.

PREFEITURA MUNICIPAL DE SÃO JOSE DOS CAMPOS. PMSB - Plano Municipal de Saneamento Básico. Disponível em: < www.sjc.sp.gov.br/media/122957/d13138_anexo01.pdf>. Acesso em 20.maio.2013.

RAMOS, Frederico Roman. Geoprocessamento Aplicado ao estudo de indicadores de desenvolvimento e fluxos migratórios no Estado de São Paulo. Disponível em < http://www.dpi.inpe.br/cursos/ser300/Trabalhos/fred.pdf $>$. Acesso em 20. Mar.2013.

THOMAS, Jefferson Angelo; BUENO, Liane da Silva;LAPOLLI, Edis Mafra. A utilização do Sensoriamento Remoto e o Geoprocessamento na Classificação de Áreas Urbanas, Joaçaba-SC. Disponível em: < http://biblioteca.cptec.inpe.br/> Acesso em 30.abr.2013 